\title{
Visit-to-Visit Variability of Lipids Measurements and the Risk of Stroke and Stroke Types: A Prospective Cohort Study
}

\author{
Anxin Wang, ${ }^{\mathrm{a}, \mathrm{b}, *}$ Haibin Li, ${ }^{\mathrm{c}, *}$ Jinhuan Yuan, ${ }^{\mathrm{d}}$ Yingting Zuo, ${ }^{\mathrm{a}, \mathrm{b}}$ Yijun Zhang, ${ }^{\mathrm{a}, \mathrm{b}}$ Shouhua Chen, \\ Shouling Wu, ${ }^{\mathrm{e}}$ Yongjun Wang ${ }^{\mathrm{a}, \mathrm{b}}$ \\ ${ }^{a}$ China National Clinical Research Center for Neurological Diseases, Beijing Tiantan Hospital, Capital Medical University, Beijing, China \\ ${ }^{b}$ Department of Neurology, Beijing Tiantan Hospital, Capital Medical University, Beijing, China \\ 'Department of Epidemiology and Health Statistics, School of Public Health, Capital Medical University, Beijing, China \\ ${ }^{d}$ Department of Epidemiology and Health Statistics, School of Public Health, North China University of Science and Technology, Tangshan, China \\ 'Department of Cardiology, Kailuan Hospital, North China University of Science and Technology, Tangshan, China
}

Background and Purpose Previous studies suggested increased visit-to-visit variability of total cholesterol (TC) is associated with stroke. This study aimed to investigate the associations of various lipids measurements variability and the risk of stroke and stroke type (ischemic and hemorrhagic stroke).

Methods Fifty-one thousand six hundred twenty participants in the Kailuan Study without history of myocardial infarction, stroke, and cancer who underwent three health examinations during 2006 to 2010 were followed for incident stroke. Variability in TC, triglycerides, high-density lipoprotein cholesterol (HDL-C), and low-density lipoprotein cholesterol (LDL-C) measurements were measured using the coefficient of variation (CV), standard deviation (SD), variability independent of the mean (VIM), and average real variability (ARV).

Results During a median of 6.04 years of follow-up, 1,189 incident stroke (1,036 ischemic and 160 hemorrhagic stroke) occurred. In the multivariable-adjusted model, the hazard ratio (HR) comparing participants in the highest versus lowest quartile of CV of HDL-C were 1.21 (95\% confidence interval $[\mathrm{Cl}], 1.02$ to 1.45 ; $P$ for trend=0.013) for ischemic stroke. The highest quartile of $\mathrm{CV}$ of LDL-C was associated with 2.17-fold risk of hemorrhagic stroke (HR, 2.17; 95\% Cl, 1.25 to 3.75 ; $P$ for trend $=0.002$ ) compared with the lowest quartile. We did not observe any significant association between TC and triglycerides variability with any of stroke. Consistent results were obtained when calculating variability index using SD, VIM, or ARV.

Conclusions These findings suggest the high visit-to-visit HDL-C and LDL-C variability were associated with an increased incidence of ischemic and hemorrhagic stroke, respectively.
Correspondence: Yongjun Wang Department of Neurology, Beijing Tiantan Hospital, Capital Medical University, No.119 South 4th Ring West Road, Fengtai District, Beijing 100070, China Tel: +86-59978538

Fax: $+86-59978538$

E-mail:yongjunwang@ncrend.org.cn

Co-correspondence: Shouling Wu Department of Cardiology, Kailuan Hospital, Hebei United University, No. 57 Xinhua East Street, Lubei District, Tangshan 063000, China. Tel: +86-03153025655 Fax: +86-03153025655 E-mail:drwusl@163.com

Received: August 7, 2019

Revised: January 9, 2020

Accepted: January 10, 2020

*These authors contributed equally to the manuscript as first author.

Keywords Stroke; Lipids; Variability; Risk factors 


\section{Introduction}

Stroke remains the leading cause of disability and mortality worldwide. ${ }^{1,2}$ Identifying and managing risk factors for stroke are effective and economic strategies for the primary prevention of stroke. ${ }^{3}$ Serum lipids levels play a crucial role in the occurrence of stroke. ${ }^{4-7}$ The associations between lipids and stroke are complex and vary by stroke subtypes. ${ }^{8-10}$ Most epidemiological cohort studies suggested lipids have the strongest associations with incident ischemic stroke relative to hemorrhagic stroke. ${ }^{9-11}$ Additionally, the associations between lipids and stroke depend on the specific lipids measurements, with strong associations for total cholesterol (TC) and low-density lipoprotein cholesterol (LDL-C). ${ }^{10,11}$ Understating the complicated relationships of lipids and stroke may be useful to prevent and treat stroke in clinical practice.

Recently, the intra-individual variability in cholesterol levels has been of increasing interest. Several clinical trials suggested that higher lipids variability predicted the adverse clinical outcomes. ${ }^{12-16}$ The American Heart Association Annual Scientific Sessions reported the findings from a post hoc analysis of the Treating to New Target (TNT) trial, suggesting that high levels of variability in LDL-C, high-density lipoprotein cholesterol (HDL-C), and triglycerides (TG) were associated with an increased risk of myocardial infarction, stroke, and mortality among patients with stable coronary artery disease. ${ }^{13}$ However, these associations were still understudied among the general population. To the best our knowledge, only one cohort study from the Korean $\mathrm{Na}$ tional Health Insurance System (NHIS) had suggested that high levels of TC variability were associated with an increased risk of stroke. ${ }^{17,18}$ Although the study was conducted in a large population, the findings needed to be further validated in depth. The associations between cholesterol and stroke risk varied in stroke subtypes, ${ }^{5}$ however, the NHIS study did not examine the impact of cholesterol variability on the risks of ischemic and hemorrhagic stroke, respectively. Additionally, the associations between variability in other lipids measurements (LDL-C, HDL-C, and TG) and risk of stroke still remained unknown.

Therefore, the aim of this study was to comprehensively investigate the associations between long-term variability in different lipids measurements over 4 years and the risks of stroke and stroke type (ischemic and hemorrhagic stroke) based on a large community-based prospective cohort study.

\section{Methods}

\section{Study design and participants}

The Kailuan Study is a prospective cohort study conducted in the Kailuan community in Tangshan City, China. ${ }^{19}$ Details of the study design have been described previously. ${ }^{19,20}$ Briefly, 101,510 participants aged 18 to 98 years were recruited in the community from June 2006 to October 2007 and underwent a comprehensive biennial health examination at the Kailuan General Hospital. Of 92,967 participants who underwent health examination in 2010 (index year), 57,927 participants underwent three health examinations from June 2006 to December 2010. We excluded 2,167 participants with missing data regarding serum lipid levels. To minimize the possible effect of reverse causality, 4,140 participants having myocardial infarction, stroke or cancer before the index year were further excluded (Figure 1). For the current analysis, 51,620 participants were included. All participants provided informed written consent. The study was performed according to the guidelines of the Helsinki Declaration and was approved by the Ethics Committee of Kailuan General Hospital (approval number: 2006-05) and Beijing Tiantan Hospital (approval number: 2010-014-01).

\section{Data collection and definitions}

Sociodemographic characteristics, lifestyles, and clinical characteristics were collected via a standard questionnaire by trained staffs, including age, sex, education, income, smoking status, drinking status, physical activity levels, and history of disease. Sex was self-reported. Educational level was categorized as illiteracy or primary school, middle school, and high school or above. The high-income level was defined as partici-

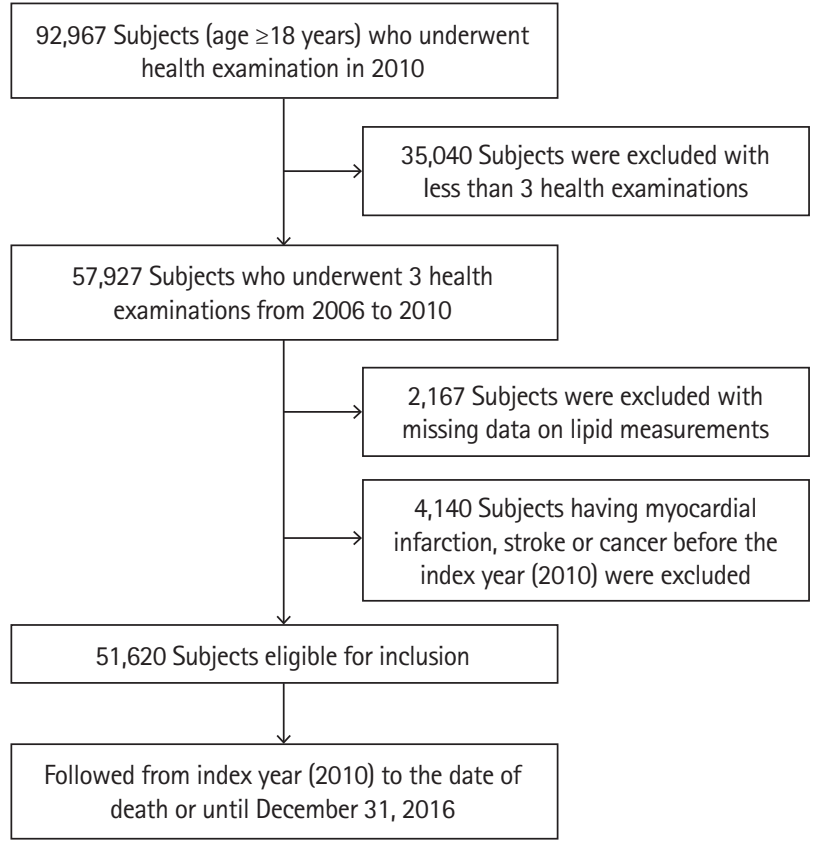

Figure 1. Flow chart of the current study. 
pants' average monthly income $>1,000$ yuan. Smoking and drinking status stratified into three levels: never, former, or current. Physical activity was evaluated based on the type and frequency of physical activity at work and during leisure time and classified as " $>4$ times per week and $>20$ minutes at a time," "<80 minutes per week," or "none." Body mass index (BMI) was calculated as weight $(\mathrm{kg}) /$ height $(\mathrm{m})^{2}$. Blood pressure was measured in the seated position using a mercury sphygmomanometer, and the average of three measurements was calculated for systolic blood pressure (SBP) and diastolic blood pressure (DBP). Hypertension was defined as SBP $\geq 140 \mathrm{~mm} \mathrm{Hg}$ or $\mathrm{DBP} \geq 90 \mathrm{~mm} \mathrm{Hg}$, any use of the antihypertensive drug, or self-reported history of hypertension.

Blood samples were collected from the antecubital vein after an overnight fast and stored in the central laboratory of the Kailuan General Hospital. All the plasma samples were measured using the Hitachi 747 auto-analyzer (Hitachi, Tokyo, Japan). Fasting blood glucose was measured with the hexokinase/ glucose-6-phosphate dehydrogenase method. Serum TC, TG, HDL-C, and LDL-C were measured with the enzymatic colorimetric method. Plasma high-sensitivity C-reactive protein (hsCRP) were measured with high-sensitivity particle-enhanced immunonephelometry assay. Serum creatinine was measured with the sarcosine oxidase assay method. The estimated glomerular filtration rate (eGFR) was calculated using the Chronic Kidney Disease Epidemiology Collaboration (CKD-EPI 2009) creatinine equation. ${ }^{21}$ Diabetes was defined as fasting blood glucose $\geq 7.0 \mathrm{mmol} / \mathrm{l}$, any use of glucose-lowering drugs, or any self-reported history of diabetes.

\section{Measures of lipids measurements variability}

Variability was defined as intra-individual variability in each serum lipids values (TC, TG, HDL-C, and LDL-C) between three visits (in years 2006, 2008, and 2010). Four indices of visit-tovisit variability that have been previously described ${ }^{13}$ were calculated, including the coefficient of variation (CV), standard deviation (SD), variability independent of the mean (VIM), and average real variability (ARV). The VIM was calculated as $100 \times$ $\mathrm{SD} /$ mean $^{\text {beta, }}$, where beta is the regression coefficient based on a natural logarithm of SD on the natural logarithm of the mean. The ARV calculated as the average absolute difference between successive measurements. The $\mathrm{CV}$ was used as the primary variability measure.

\section{Assessment of stroke}

The primary outcome was the first occurrence of stroke, either nonfatal or fatal. Participants were followed up by face-toface interviews at every 2-year routine medical examination until December 31, 2016. The outcome information was further confirmed by checking discharge summaries from the 11 hospitals and medical records from medical insurance. For the participants without face-to-face follow-ups, outcome information was obtained directly by checking death certificates from provincial vital statistics offices, discharge summaries, and medical records. Stroke was diagnosed according to the World Health Organization criteria ${ }^{22}$ combined with brain computed tomography or magnetic resonance imaging (MRI) for confirmation. Stroke was classified into two main subtypes: ischemic and hemorrhagic stroke.

\section{Statistical analysis}

Baseline characteristics are presented as the mean \pm standard deviation or percentage. Differences between incident stroke and non-stroke participants were compared using Student's ttest or Mann-Whitney $U$ test for continuous variables and Pearson's chi-sqaure test for categorical variables.

Person-years was calculated from the index year (2010) to the first occurrence of stroke, mortality, or the end of the study (December 31, 2016), whichever came first. The incidence rate of stroke was calculated by dividing the number of incident cases by the total follow-up duration (person-years). We used Cox proportional hazards regression models to estimate the association between each of serum lipids variability (TC, TG, HDL-C, and LDL-C) and incident stroke (total, ischemic, and hemorrhagic stroke) in separate analyses. Serum lipids variability categorized in quartiles and also modelled as a continuous variable in the analyses. Proportional hazards assumption was satisfied by checking the Schoenfeld residual plots. To adjust for potential confounding factors, three models were built as follows: Model 1, minimally adjusted for sex and age; Model 2 , adjusted for sex, age, use of lipid-lowering agent, and mean serum specific lipids levels (e.g., adjusted for mean TC concentration when analyses for TC variability); Model 3 further adjusted for education, income, smoking status, drinking status, physical activity, diabetes, hypertension, BMI, eGFR, and hsCRP. The hazard ratio (HR) and 95\% confidence interval (CI) were reported. $P$-values for trend were computed using quartiles as ordinal variables.

To evaluate the stability of our main results, sensitivity analyses was conducted by excluding the participants who used cholesterol-lowering agents to explore whether the potential each of lipids variability-stroke relation was confounded by cholesterol-lowering agents (e.g., statins). All analyses were conducted using SAS version 9.4 (SAS Institute Inc., Cary, NC, USA). A two-sided $P<0.05$ was considered statistically significant. 
Table 1. Baseline characteristics according to incident stroke status

\begin{tabular}{|c|c|c|c|c|}
\hline Characteristic & $\begin{array}{c}\text { Total } \\
(n=51,620)\end{array}$ & $\begin{array}{l}\text { Incident stroke } \\
\quad(n=1,189)\end{array}$ & $\begin{array}{l}\text { No incident stroke } \\
\quad(n=50,431)\end{array}$ & $P$ \\
\hline Age (yr) & $52.79 \pm 11.80$ & $60.06 \pm 9.99$ & $52.61 \pm 11.79$ & $<0.001$ \\
\hline Male sex (\%) & 75.95 & 87.80 & 75.67 & $<0.001$ \\
\hline High school or above (\%) & 28.08 & 13.16 & 28.44 & $<0.001$ \\
\hline Current smoker (\%) & 33.78 & 36.62 & 33.71 & 0.036 \\
\hline Current drinker (\%) & 34.98 & 35.22 & 34.97 & 0.860 \\
\hline Physical activity (> 4 times/week) (\%) & 14.22 & 16.12 & 14.18 & 0.059 \\
\hline High income (> ¥1,000/month) (\%) & 45.85 & 43.04 & 45.92 & 0.051 \\
\hline Hypertension (\%) & 44.36 & 73.25 & 43.68 & $<0.001$ \\
\hline Diabetes (\%) & 10.28 & 21.78 & 10.01 & $<0.001$ \\
\hline On lipid-lowering agent (\%) & 1.54 & 2.02 & 1.52 & 0.170 \\
\hline Body mass index $\left(\mathrm{kg} / \mathrm{m}^{2}\right)$ & $25.09 \pm 3.38$ & $25.69 \pm 3.39$ & $25.08 \pm 3.38$ & $<0.001$ \\
\hline Systolic BP (mm Hg) & $130.42 \pm 19.09$ & $143.52 \pm 20.44$ & $130.11 \pm 18.95$ & $<0.001$ \\
\hline Diastolic BP (mm Hg) & $84.15 \pm 10.79$ & $90.03 \pm 11.64$ & $84.01 \pm 10.73$ & $<0.001$ \\
\hline Fasting blood glucose (mmol/L) & $5.63 \pm 1.71$ & $6.23 \pm 2.19$ & $5.62 \pm 1.70$ & $<0.001$ \\
\hline eGFR (mL/min/1.73 m²) & $90.65 \pm 19.63$ & $85.09 \pm 19.45$ & $90.78 \pm 19.62$ & $<0.001$ \\
\hline hs-CRP (mg/L) & $2.32 \pm 5.07$ & $3.00 \pm 6.44$ & $2.31 \pm 5.04$ & $<0.001$ \\
\hline \multicolumn{5}{|l|}{ Total cholesterol (mmol/L) } \\
\hline Baseline & $4.98 \pm 0.97$ & $5.15 \pm 0.99$ & $4.98 \pm 0.97$ & $<0.001$ \\
\hline Mean & $4.96 \pm 0.83$ & $5.12 \pm 0.85$ & $4.96 \pm 0.82$ & $<0.001$ \\
\hline SD & $0.55 \pm 0.40$ & $0.57 \pm 0.41$ & $0.55 \pm 0.40$ & 0.130 \\
\hline $\mathrm{CV}$ & $11.34 \pm 8.81$ & $11.36 \pm 8.80$ & $11.34 \pm 8.81$ & 0.950 \\
\hline VIM & $0.56 \pm 0.40$ & $0.56 \pm 0.41$ & $0.56 \pm 0.41$ & 0.470 \\
\hline ARV & $0.68 \pm 0.51$ & $0.71 \pm 0.52$ & $0.68 \pm 0.51$ & 0.065 \\
\hline \multicolumn{5}{|l|}{ Triglycerides (mmol/L) } \\
\hline Baseline & $1.66 \pm 1.42$ & $1.83 \pm 1.68$ & $1.66 \pm 1.41$ & $<0.001$ \\
\hline Mean & $1.65 \pm 1.11$ & $1.81 \pm 1.23$ & $1.64 \pm 1.11$ & $<0.001$ \\
\hline SD & $0.60 \pm 0.78$ & $0.66 \pm 0.87$ & $0.60 \pm 0.78$ & 0.003 \\
\hline CV & $31.89 \pm 20.39$ & $31.79 \pm 20.88$ & $31.89 \pm 20.38$ & 0.870 \\
\hline VIM & $0.54 \pm 0.32$ & $0.52 \pm 0.31$ & $0.54 \pm 0.32$ & 0.046 \\
\hline ARV & $0.74 \pm 1.00$ & $0.81 \pm 1.11$ & $0.73 \pm 0.99$ & 0.010 \\
\hline \multicolumn{5}{|l|}{$\mathrm{HDL}-\mathrm{C}(\mathrm{mmol} / \mathrm{L})$} \\
\hline Baseline & $1.56 \pm 0.45$ & $1.53 \pm 0.46$ & $1.56 \pm 0.45$ & 0.015 \\
\hline Mean & $1.54 \pm 0.30$ & $1.53 \pm 0.32$ & $1.54 \pm 0.30$ & 0.480 \\
\hline SD & $0.29 \pm 0.20$ & $0.30 \pm 0.22$ & $0.28 \pm 0.20$ & 0.024 \\
\hline $\mathrm{CV}$ & $18.31 \pm 11.26$ & $19.30 \pm 12.14$ & $18.29 \pm 11.24$ & 0.002 \\
\hline VIM & $0.28 \pm 0.18$ & $0.30 \pm 0.19$ & $0.28 \pm 0.18$ & 0.001 \\
\hline ARV & $0.34 \pm 0.26$ & $0.36 \pm 0.29$ & $0.34 \pm 0.26$ & 0.113 \\
\hline \multicolumn{5}{|l|}{ LDL-C (mmol/L) } \\
\hline Baseline & $2.59 \pm 0.79$ & $2.66 \pm 0.89$ & $2.59 \pm 0.78$ & 0.001 \\
\hline Mean & $2.49 \pm 0.62$ & $2.53 \pm 0.66$ & $2.48 \pm 0.62$ & 0.019 \\
\hline SD & $0.56 \pm 0.39$ & $0.64 \pm 0.45$ & $0.56 \pm 0.38$ & $<0.001$ \\
\hline
\end{tabular}


Table 1. Continued

\begin{tabular}{|c|c|c|c|c|}
\hline Characteristic & $\begin{array}{c}\text { Total } \\
(n=51,620)\end{array}$ & $\begin{array}{c}\text { Incident stroke } \\
(\mathrm{n}=1,189)\end{array}$ & $\begin{array}{l}\text { No incident stroke } \\
\quad(n=50,431)\end{array}$ & $P$ \\
\hline CV & $24.15 \pm 18.87$ & $27.55 \pm 21.83$ & $24.07 \pm 18.78$ & $<0.001$ \\
\hline VIM & $0.56 \pm 0.39$ & $0.64 \pm 0.45$ & $0.56 \pm 0.38$ & $<0.001$ \\
\hline ARV & $0.70 \pm 0.55$ & $0.79 \pm 0.63$ & $0.70 \pm 0.55$ & $<0.001$ \\
\hline
\end{tabular}

Values are presented as mean \pm standard deviation or percentage.

$\mathrm{BP}$, blood pressure; eGFR, estimated glomerular filtration rate; hs-CRP, high-sensitivity C-reactive protein; $\mathrm{SD}$, standard deviation; CV, coefficient of variation; VIM, variability independent of the mean; ARV, average real variability; HDL-C, high-density lipoprotein cholesterol; LDL-C, low-density lipoproteins cholesterol.

Table 2. Hazard ratios and 95\% confidence intervals of incident stroke by quartiles of total cholesterol variability (coefficient of variation)

\begin{tabular}{|c|c|c|c|c|c|c|}
\hline Variable & Quartile 1 & Quartile 2 & Quartile 3 & Quartile 4 & Per-SD increase & $P_{\text {trend }}$ \\
\hline \multicolumn{7}{|l|}{ Total stroke } \\
\hline No. of cases & 307 & 283 & 299 & 300 & & \\
\hline Person-years & $75,757.31$ & $75,638.51$ & $75,348.25$ & $75,059.45$ & & \\
\hline Incidence rate ${ }^{*}$ & 4.05 & 3.74 & 3.97 & 4.00 & & \\
\hline Model 1 & 1.00 & $0.94(0.80-1.10)$ & $1.02(0.87-1.20)$ & $1.06(0.90-1.25)$ & $1.03(0.97-1.09)$ & 0.299 \\
\hline Model 2 & 1.00 & $0.93(0.79-1.10)$ & $1.03(0.87-1.20)$ & $1.08(0.92-1.27)$ & $1.05(0.99-1.12)$ & 0.218 \\
\hline Model 3 & 1.00 & $0.93(0.79-1.10)$ & $1.03(0.87-1.21)$ & $1.06(0.90-1.25)$ & $1.03(0.97-1.09)$ & 0.334 \\
\hline Sensitivity analyses $^{\dagger}$ & 1.00 & $0.95(0.80-1.12)$ & $1.05(0.89-1.23)$ & $1.05(0.88-1.24)$ & $1.04(0.98-1.11)$ & 0.411 \\
\hline \multicolumn{7}{|l|}{ Ischemic stroke } \\
\hline No. of cases & 262 & 247 & 257 & 270 & & \\
\hline Person-years & $75,854.72$ & $75,708.08$ & $75,445.74$ & $75,124.03$ & & \\
\hline Incidence rate ${ }^{*}$ & 3.45 & 3.26 & 3.41 & 3.59 & & \\
\hline Model 1 & 1.00 & $0.96(0.81-1.41)$ & $1.03(0.87-1.23)$ & $1.12(0.95-1.33)$ & $1.04(0.98-1.10)$ & 0.125 \\
\hline Model 2 & 1.00 & $0.96(0.80-1.14)$ & $1.03(0.87-1.23)$ & $1.15(0.97-1.36)$ & $1.07(1.01-1.14)$ & 0.080 \\
\hline Model 3 & 1.00 & $0.96(0.80-1.15)$ & $1.03(0.86-1.23)$ & $1.12(0.94-1.34)$ & $1.06(1.00-1.13)$ & 0.149 \\
\hline Sensitivity analyses ${ }^{+}$ & 1.00 & $0.97(0.81-1.16)$ & $1.05(0.88-1.25)$ & $1.10(0.91-1.31)$ & $1.06(0.99-1.13)$ & 0.234 \\
\hline \multicolumn{7}{|l|}{ Hemorrhagic stroke } \\
\hline No. of cases & 46 & 39 & 42 & 33 & & \\
\hline Person-years & $76,399.33$ & $76,211.17$ & $75,952.59$ & $75,689.55$ & & \\
\hline Incidence rate ${ }^{*}$ & 0.60 & 0.51 & 0.55 & 0.44 & & \\
\hline Model 1 & 1.00 & $0.86(0.56-1.32)$ & $0.95(0.63-1.44)$ & $0.77(0.49-1.20)$ & $0.94(0.79-1.11)$ & 0.341 \\
\hline Model 2 & 1.00 & $0.86(0.56-1.32)$ & $0.95(0.62-1.44)$ & $0.76(0.49-1.20)$ & $0.94(0.79-1.11)$ & 0.329 \\
\hline Model 3 & 1.00 & $0.83(0.53-1.29)$ & $0.92(0.60-1.42)$ & $0.71(0.45-1.14)$ & $0.89(0.74-1.07)$ & 0.236 \\
\hline Sensitivity analyses $^{+}$ & 1.00 & $0.87(0.55-1.36)$ & $0.95(0.60-1.47)$ & $0.74(0.46-1.19)$ & $0.89(0.74-1.08)$ & 0.298 \\
\hline
\end{tabular}

Model 1 adjusted for sex and age; Model 2 adjusted for sex, age, mean total cholesterol level, and use of lipid-lowering agent; Model 3 adjusted for covariables in Model 2 plus education, income, smoking status, drinking status, physical activity, diabetes, hypertension, body mass index, estimated glomerular filtration rate, and high-sensitivity C-reactive protein.

$\mathrm{SD}$, standard deviation.

${ }^{*}$ Incidence rate per 1,000 person-years; ${ }^{+}$Excluded participants with use of lipid-lowering agent and adjusted for covariables in Model 3.

\section{Data availability}

Data are available to researchers on request by contacting with corresponding authors (Dr. Wu or Dr. Wang).

\section{Results}

The final sample included 51,620 participants. The mean age of the population was $52.79 \pm 11.80$ years. During a median follow-up of 6.04 years, 1,189 strokes occurred. There were 1,036 incident cases of ischemic stroke, and 160 cases of hemor- 

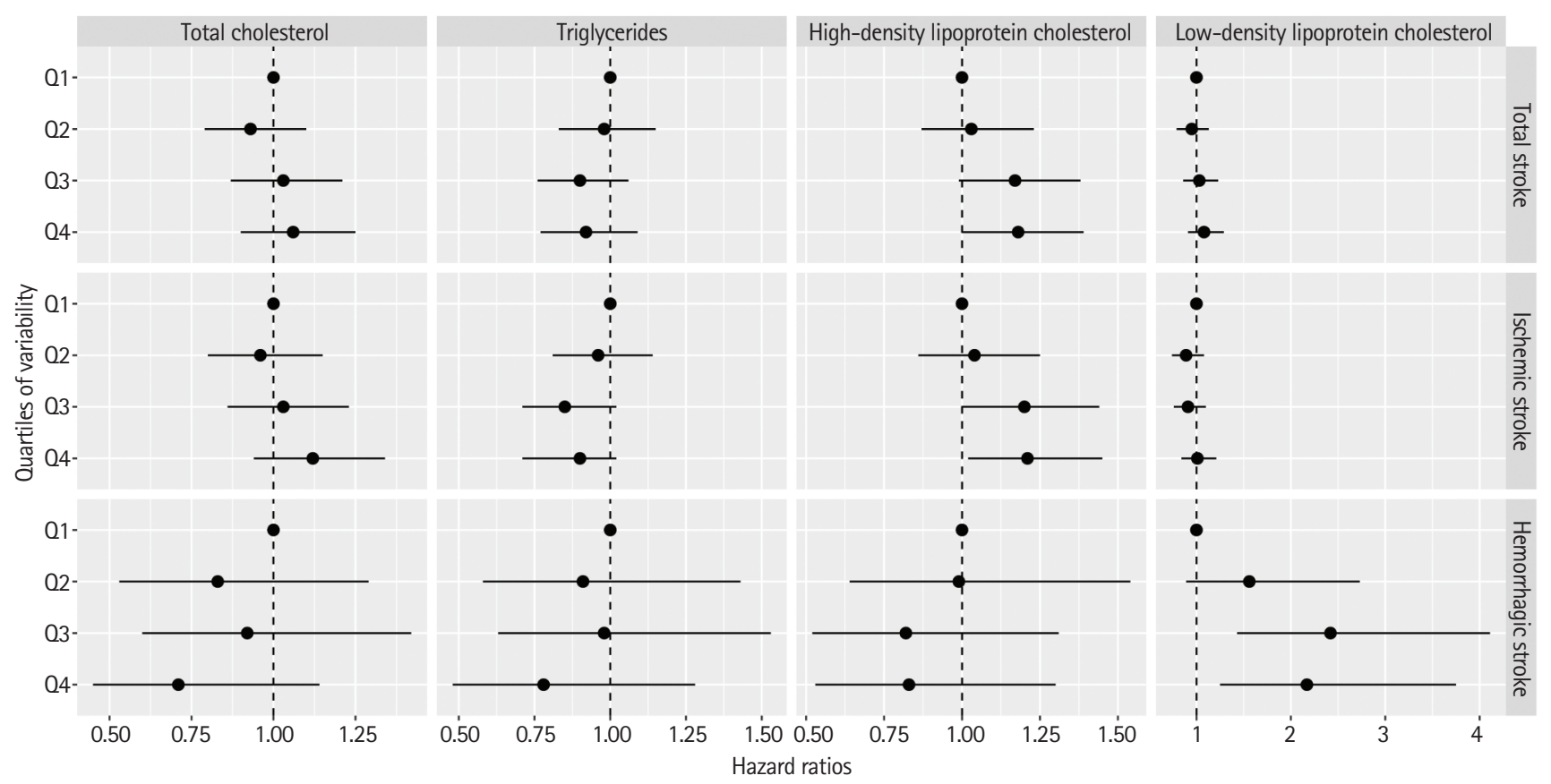

Figure 2. The hazard ratio and 95\% confidence interval of total, ischemic and hemorrhagic stroke by quartiles of total cholesterol, triglycerides, high-density lipoprotein cholesterol, and low-density lipoprotein cholesterol variability measured using the coefficient of variation in the fully adjusted model (Model 3 ).

rhagic stroke. The incidence rate of stroke was 3.96 per 1,000 person-years. Baseline characteristics of the study population are presented in Table 1. There was a significant difference between the incident stroke and non-stroke group in age, sex, education, smoking, hypertension, diabetes, eGFR, hs-CRP, baseline TC, TG, HDL-C, and LDL-C. The variability of HDL-C and LDL-C were significantly higher in participants with incident stroke than in those without stroke.

We did not observe any significant association between TC (Table 2 and Figure 2) or TG (Table 3 and Figure 2) variability with any risk of total, ischemic, and hemorrhagic stroke. However, in the fully adjusted model (Model 3), when comparing the highest quartile to the lowest quartile of HDL-C variability, HDL-C variability was significantly positively associated with total stroke ( $\mathrm{HR}, 1.18 ; 95 \% \mathrm{Cl}, 1.00$ to $1.39 ; P$ for trend=0.024), ischemic stroke (HR, 1.21; $95 \% \mathrm{Cl}, 1.02$ to $1.45 ; P$ for trend=0.013), but not with hemorrhagic stroke $(\mathrm{HR}, 0.83 ; 95 \% \mathrm{Cl}, 0.53$ to $1.30 ; P$ for trend $=0.308$ ) (Table 4 and Figure 2). For the highest quartile in LDL-C variability compared with the lowest quartile, the risk of hemorrhagic stroke increased by $117 \%(\mathrm{HR}, 2.17 ; 95 \% \mathrm{Cl}$, 1.25 to $3.75 ;$; for trend=0.002) (Table 5 and Figure 2). Very similar results were observed when variability measured using SD, VIM, or ARV (Supplementary Tables 1-4).

After excluding the participants who used cholesterol-lowering agents, the highest quartile of LDL-C variability was associated with 2.30-fold risk of hemorrhagic stroke (HR, 2.30; 95\%
$\mathrm{Cl}, 1.32$ to 4.02 ; $P$ for trend=0.001) (Table 5). Additionally, for a per-SD increase in LDL-C variability, the fully adjusted HR of hemorrhagic stroke was $1.24(95 \% \mathrm{Cl}, 1.06$ to 1.43$)$ (Table 5).

\section{Discussion}

In this large community-based cohort study, we found that high variability in certain lipid measurements were associated with elevated risk of stroke and stroke type. High variability in LDL-C and HDL-C concentrations were associated with a higher risk for ischemic stroke and hemorrhagic stroke during a median follow-up of 6.04 years, respectively. These associations persisted after multivariable adjustment for important potential confounders, including traditional cardiometabolic risk factors, use of lipid-lowering agent, mean LDL-C, and HDL-C concentrations in separate models.

The visit-to-visit variability in various biological measures has been of increasing interest in recent years. For example, higher variability in blood pressure, glucose, cholesterol, and body weight is independently associated with a wide range of health outcomes, such as cardiovascular events, diabetes, endstage renal disease, dementia, and all-cause mortality. . $^{12-14,18,23-28}$ Until now, to our knowledge, only two randomized controlled trials, $_{1}{ }^{12-14}$ and one population-based cohort study ${ }^{17,18}$ suggested that cholesterol variability is an independent predictor of stroke. Our current study further revealed the relationship be- 
Table 3. Hazard ratios and 95\% confidence intervals of incident stroke by quartiles of triglycerides variability (coefficient of variation)

\begin{tabular}{|c|c|c|c|c|c|c|}
\hline Variable & Quartile 1 & Quartile 2 & Quartile 3 & Quartile 4 & Per-SD increase & $P_{\text {trend }}$ \\
\hline \multicolumn{7}{|l|}{ Total stroke } \\
\hline No. of cases & 315 & 307 & 281 & 286 & & \\
\hline Person-years & $75,245.63$ & $75,164.56$ & $75,303.85$ & $76,089.49$ & & \\
\hline Incidence rate ${ }^{*}$ & 4.19 & 4.08 & 3.73 & 3.76 & & \\
\hline Model 1 & 1.00 & $1.01(0.87-1.19)$ & $0.96(0.82-1.13)$ & $1.06(0.90-1.25)$ & $1.05(1.00-1.12)$ & 0.647 \\
\hline Model 2 & 1.00 & $1.00(0.87-1.17)$ & $0.93(0.79-1.09)$ & $0.94(0.79-1.11)$ & $1.00(0.94-1.06)$ & 0.320 \\
\hline Model 3 & 1.00 & $0.98(0.83-1.15)$ & $0.90(0.76-1.06)$ & $0.92(0.77-1.09)$ & $1.00(0.94-1.06)$ & 0.206 \\
\hline Sensitivity analyses $^{\dagger}$ & 1.00 & $0.99(0.84-1.17)$ & $0.90(0.76-1.07)$ & $0.94(0.79-1.11)$ & $1.00(0.94-1.07)$ & 0.286 \\
\hline \multicolumn{7}{|l|}{ Ischemic stroke } \\
\hline No. of cases & 281 & 266 & 237 & 252 & & \\
\hline Person-years & $7,5291.21$ & $7,5259.64$ & $75,414.32$ & $76,167.40$ & & \\
\hline Incidence rate ${ }^{*}$ & 3.73 & 3.53 & 3.14 & 3.31 & & \\
\hline Model 1 & 1.00 & $0.98(0.83-1.16)$ & $0.91(0.76-1.08)$ & $1.05(0.89-1.25)$ & $1.05(0.99-1.12)$ & 0.849 \\
\hline Model 2 & 1.00 & $0.97(0.82-1.15)$ & $0.87(0.74-1.04)$ & $0.91(0.76-1.09)$ & $0.98(0.93-1.05)$ & 0.175 \\
\hline Model 3 & 1.00 & $0.96(0.81-1.14)$ & $0.85(0.71-1.02)$ & $0.90(0.71-1.02)$ & $0.99(0.92-1.06)$ & 0.129 \\
\hline Sensitivity analyses ${ }^{+}$ & 1.00 & $0.98(0.82-1.17)$ & $0.86(0.72-1.03)$ & $0.92(0.76-1.10)$ & $1.00(0.93-1.06)$ & 0.181 \\
\hline \multicolumn{7}{|l|}{ Hemorrhagic stroke } \\
\hline No. of cases & 41 & 42 & 44 & 33 & & \\
\hline Person-years & $75,847.28$ & $75,789.63$ & $75,882.09$ & $76,733.64$ & & \\
\hline Incidence rate* & 0.54 & 0.55 & 0.58 & 0.43 & & \\
\hline Model 1 & 1.00 & $1.05(0.68-1.62)$ & $1.13(0.74-1.73)$ & $0.90(0.57-1.43)$ & $0.99(0.84-1.16)$ & 0.800 \\
\hline Model 2 & 1.00 & $1.05(0.68-1.62)$ & $1.13(0.74-1.74)$ & $0.90(0.56-1.45)$ & $1.00(0.84-1.18)$ & 0.825 \\
\hline Model 3 & 1.00 & $0.91(0.58-1.43)$ & $0.98(0.63-1.53)$ & $0.78(0.48-1.28)$ & $0.96(0.80-1.15)$ & 0.428 \\
\hline Sensitivity analyses $^{+}$ & 1.00 & $0.91(0.57-1.43)$ & $0.95(0.61-1.49)$ & $0.80(0.49-1.30)$ & $0.96(0.80-1.16)$ & 0.436 \\
\hline
\end{tabular}

Model 1 adjusted for sex and age; Model 2 adjusted for sex, age, mean triglycerides level, and use of lipid-lowering agent; Model 3 adjusted for covariables in Model 2 plus education, income, smoking status, drinking status, physical activity, diabetes, hypertension, body mass index, estimated glomerular filtration rate, and high-sensitivity C-reactive protein.

${ }^{*}$ Incidence rate per 1,000 person-years; ${ }^{\dagger}$ Excluded participants with use of lipid-lowering agent and adjusted for covariables in Model 3.

tween different lipids measurements variability and incident stroke and stroke subtypes.

A previous review suggested that most but not all large prospective cohort studies revealed higher baseline TC are linked to increased ischemic stroke risk. ${ }^{29}$ On the contrary, an additional meta-analysis of 23 studies showed an inverse relationship between baseline TC and hemorrhagic stroke. ${ }^{6}$ However, the relationship between TC and stroke may be biased because TC concentrations were only measured at baseline. In the general population, the relationship between TC variability and incident stroke is first reported in the NHIS study. The NHIS study included 3,650,000 Koreans who had at least three TC measurements from January 2002 to December 2007 and suggested that participants in the highest quartiles of TC variability using CV had 11\% higher risk of stroke compared with the lowest quartiles ( $\mathrm{HR}, 1.11 ; 95 \% \mathrm{Cl}, 1.08$ to 1.14) during a median follow-up of 8.3 years. ${ }^{17}$ In contrast to the NHIS study, these associations became nonsignificant after further adjustment of mean TC concentrations and use of lipid-lowering agent. This difference may be contributed to the differing patterns of stroke risk factor profiles between Koreans and Chinese. ${ }^{30}$

High levels of TG are considered to be risk factors for stroke. ${ }^{31}$ Few studies had reported the associations between TG variability and stroke risk. A post hoc analysis of the TNT trial with 9,572 patients aged 35 to 75 years suggested that high variability of TG was associated with 66\% increased risk of incident stroke (HR, 1.66; 95\% Cl, 1.16 to 2.36 for the highest vs. lowest quartiles) after adjusted for baseline traditional risk factors, mean TG concentrations, and use of lipid-lowering agent..$^{13}$ The mechanisms that mediate the link between TG variability and stroke are not clear. As reported previously, high TG variability was associated with incident microalbuminuria, ${ }_{1}^{32}$ and renal dysfunction, which is a strong predeictor of incident stroke in previous studies conducted in Kailuan Study. ${ }^{33,34}$ Further investigations are required to understand the relationship 
Table 4. Hazard ratios and 95\% confidence intervals of incident stroke by quartiles of high-density lipoprotein cholesterol variability (coefficient of variation)

\begin{tabular}{|c|c|c|c|c|c|c|}
\hline Variable & Quartile 1 & Quartile 2 & Quartile 3 & Quartile 4 & Per-SD increase & $P_{\text {trend }}$ \\
\hline \multicolumn{7}{|l|}{ Total stroke } \\
\hline No. of cases & 285 & 268 & 307 & 329 & & \\
\hline Person-years & $75,180.70$ & $75,595.96$ & $75,430.04$ & $75,596.81$ & & \\
\hline Incidence rate ${ }^{*}$ & 3.79 & 3.55 & 4.07 & 4.35 & & \\
\hline Model 1 & 1.00 & $0.98(0.83-1.16)$ & $1.16(0.99-1.36)$ & $1.19(1.01-1.39)$ & $1.08(1.02-1.14)$ & 0.009 \\
\hline Model 2 & 1.00 & $0.99(0.84-1.17)$ & $1.16(0.99-1.37)$ & $1.19(1.02-1.40)$ & $1.08(1.03-1.14)$ & 0.007 \\
\hline Model 3 & 1.00 & $1.03(0.87-1.23)$ & 1.17 (0.99-1.38) & $1.18(1.00-1.39)$ & $1.06(1.01-1.12)$ & 0.024 \\
\hline Sensitivity analyses ${ }^{+}$ & 1.00 & $1.03(0.87-1.22)$ & $1.14(0.96-1.35)$ & $1.17(0.99-1.38)$ & $1.06(1.01-1.12)$ & 0.041 \\
\hline \multicolumn{7}{|l|}{ Ischemic stroke } \\
\hline No. of cases & 244 & 232 & 269 & 291 & & \\
\hline Person-years & $75,263.68$ & $75,666.67$ & $75,539.08$ & $75,663.15$ & & \\
\hline Incidence rate ${ }^{*}$ & 3.24 & 3.07 & 3.56 & 3.85 & & \\
\hline Model 1 & 1.00 & $1.00(0.83-1.19)$ & $1.19(1.00-1.41)$ & $1.22(1.03-1.45)$ & $1.09(1.03-1.15)$ & 0.005 \\
\hline Model 2 & 1.00 & $1.00(0.83-1.20)$ & $1.19(1.00-1.42)$ & $1.24(1.04-1.47)$ & $1.10(1.04-1.16)$ & 0.004 \\
\hline Model 3 & 1.00 & $1.04(0.86-1.25)$ & $1.20(1.00-1.44)$ & $1.21(1.02-1.45)$ & $1.10(1.02-1.14)$ & 0.013 \\
\hline Sensitivity analyses $^{+}$ & 1.00 & $1.04(0.86-1.25)$ & $1.17(0.97-1.40)$ & $1.20(1.00-1.43)$ & $1.07(1.01-1.14)$ & 0.025 \\
\hline \multicolumn{7}{|l|}{ Hemorrhagic stroke } \\
\hline No. of cases & 45 & 40 & 37 & 38 & & \\
\hline Person-years & 75770.39 & 76134.28 & 76084.01 & 76263.97 & & \\
\hline Incidence rate ${ }^{*}$ & 0.59 & 0.53 & 0.49 & 0.50 & & \\
\hline Model 1 & 1.00 & $0.92(0.60-1.41)$ & $0.87(0.56-1.34)$ & $0.86(0.56-1.33)$ & $0.96(0.82-1.12)$ & 0.474 \\
\hline Model 2 & 1.00 & $0.92(0.60-1.41)$ & $0.86(0.56-1.33)$ & $0.85(0.55-1.32)$ & $0.95(0.82-1.12)$ & 0.437 \\
\hline Model 3 & 1.00 & $0.99(0.64-1.54)$ & $0.82(0.52-1.31)$ & $0.83(0.53-1.30)$ & $0.92(0.78-1.08)$ & 0.308 \\
\hline Sensitivity analyses $^{+}$ & 1.00 & $0.98(0.63-1.53)$ & $0.81(0.50-1.30)$ & $0.81(0.51-1.29)$ & $0.91(0.77-1.08)$ & 0.281 \\
\hline
\end{tabular}

Model 1 adjusted for sex and age; Model 2 adjusted for sex, age, mean high-density lipoprotein cholesterol level, and use of lipid-lowering agent; Model 3 adjusted for covariables in Model 2 plus education, income, smoking status, drinking status, physical activity, diabetes, hypertension, body mass index, estimated glomerular filtration rate, and high-sensitivity C-reactive protein.

*Incidence rate per 1,000 person-years; ${ }^{\dagger}$ Excluded participants with use of lipid-lowering agent and adjusted for covariables in Model 3.

of TG variability, stroke and its subtypes.

Studies on the associations of HDL-C and LDL-C variability and risks of stroke are limited. As noted previously in TNT trial, for every 1-SD increase in HDL-C and LDL-C variability, the risk of stroke increased by $17 \%(\mathrm{HR}, 1.17 ; 95 \% \mathrm{Cl}, 1.12$ to 1.22$)$ and $14 \%(\mathrm{HR}, 1.14 ; 95 \% \mathrm{Cl}, 1.01$ to 1.26$)$ among patients with stable coronary artery disease, respectively. ${ }^{12,13}$ In a cohort study of 130 patients with ST-segment elevation myocardial infarction (STEMI), a higher HDL-C and LDL-C variability was associated with a higher risk of 5 -year of major adverse cardiac event, including death, myocardial infarction, stroke, unplanned revascularization, and heart failure admission. ${ }^{14}$ We similarly observed that high variability of HDL-C and LDL-C were respectively associated with an increased incidence of hemorrhagic and ischemic stroke independent of use of lipid-lowering agent, mean HDL-C or LDL-C concentrations in separate models.

The potential biologically plausible mechanisms underlying our findings remain unknown, but there are several hypotheses. First, the greater visit-to-visit variability in HDL-C and LDL-C is linked with the percent atheroma volume progression, which is an independent risk factor for stroke. ${ }^{16}$ Second, a higher LDL-C variability is associated with lower cerebral blood flow and greater white matter hyperintensity on brain MRI. ${ }^{15}$ Third, lipid variability is an epiphenomenon of pathologic conditions (e.g., frailty) that increase the risk of stroke. ${ }^{12}$ Further research is still required to interpret why high variability of HDL-C and LDL-C is associated with different type of stroke.

The strengths of this study include its prospective design, the large population with a complete follow-up of stroke and stroke subtypes, and repeated assessment of various lipids measurements. However, our study has several limitations. The first limitation of this study is that we measured lipids within the first three waves and did not investigate long-term variability (e.g., 10 years) in lipids value. This design was chosen in 
Table 5. Hazard ratios and 95\% confidence intervals of incident stroke by quartiles of low-density lipoprotein cholesterol variability (coefficient of variation)

\begin{tabular}{|c|c|c|c|c|c|c|}
\hline Variable & Quartile 1 & Quartile 2 & Quartile 3 & Quartile 4 & Per-SD increase & $P_{\text {trend }}$ \\
\hline \multicolumn{7}{|l|}{ Total stroke } \\
\hline No. of cases & 273 & 257 & 286 & 373 & & \\
\hline Person-years & $76,169.51$ & $75,523.07$ & $74,991.67$ & $75,119.27$ & & \\
\hline Incidence rate ${ }^{*}$ & 3.58 & 3.40 & 3.81 & 4.97 & & \\
\hline Model 1 & 1.00 & $0.96(0.81-1.14)$ & $1.02(0.86-1.21)$ & $1.10(0.94-1.29)$ & $1.05(1.00-1.11)$ & 0.160 \\
\hline Model 2 & 1.00 & $0.97(0.82-1.15)$ & $1.05(0.89-1.24)$ & $1.19(1.00-1.40)$ & $1.09(1.04-1.15)$ & 0.028 \\
\hline Model 3 & 1.00 & $0.95(0.79-1.13)$ & $1.03(0.86-1.23)$ & $1.08(0.91-1.29)$ & $1.06(1.00-1.12)$ & 0.265 \\
\hline Sensitivity analyses $^{\dagger}$ & 1.00 & $0.93(0.78-1.12)$ & $1.03(0.86-1.23)$ & $1.09(0.91-1.30)$ & $1.06(1.00-1.12)$ & 0.238 \\
\hline \multicolumn{7}{|l|}{ Ischemic stroke } \\
\hline No. of cases & 250 & 224 & 238 & 324 & & \\
\hline Person-years & $76,220.08$ & $75,590.64$ & $75,113.27$ & $75,208.58$ & & \\
\hline Incidence rate ${ }^{*}$ & 3.28 & 2.96 & 3.17 & 4.31 & & \\
\hline Model 1 & 1.00 & $0.92(0.77-1.10)$ & $0.93(0.78-1.11)$ & $1.04(0.88-1.23)$ & $1.03(0.98-1.09)$ & 0.570 \\
\hline Model 2 & 1.00 & $0.93(0.78-1.11)$ & $0.96(0.80-1.14)$ & $1.13(0.95-1.34)$ & $1.08(1.01-1.14)$ & 0.162 \\
\hline Model 3 & 1.00 & $0.89(0.74-1.08)$ & $0.91(0.76-1.10)$ & $1.01(0.84-1.21)$ & $1.03(0.97-1.10)$ & 0.864 \\
\hline Sensitivity analyses ${ }^{+}$ & 1.00 & $0.88(0.73-1.06)$ & $0.91(0.75-1.10)$ & $1.00(0.83-1.21)$ & $1.03(0.97-1.10)$ & 0.876 \\
\hline \multicolumn{7}{|l|}{ Hemorrhagic stroke } \\
\hline No. of cases & 24 & 35 & 49 & 52 & & \\
\hline Person-years & $76,767.95$ & $76,078.85$ & $75,551.14$ & $75,854.71$ & & \\
\hline Incidence rate* & 0.31 & 0.46 & 0.65 & 0.69 & & \\
\hline Model 1 & 1.00 & $1.49(0.88-2.50)$ & $2.02(1.24-3.29)$ & $1.86(1.14-3.04)$ & $1.18(1.04-1.34)$ & 0.008 \\
\hline Model 2 & 1.00 & $1.50(0.89-2.52)$ & $2.05(1.26-3.36)$ & $1.96(1.18-3.25)$ & $1.23(1.07-1.41)$ & 0.005 \\
\hline Model 3 & 1.00 & $1.56(0.89-2.73)$ & $2.42(1.43-4.11)$ & $2.17(1.25-3.75)$ & $1.22(1.05-1.41)$ & 0.002 \\
\hline Sensitivity analyses $^{+}$ & 1.00 & $1.59(0.90-2.82)$ & $2.52(1.47-4.32)$ & $2.30(1.32-4.02)$ & $1.24(1.06-1.43)$ & 0.001 \\
\hline
\end{tabular}

Model 1 adjusted for sex and age; Model 2 adjusted for sex, age, mean low-density lipoprotein cholesterol, and use of lipid-lowering agent; Model 3 adjusted for covariables in Model 2 plus education, income, smoking status, drinking status, physical activity, diabetes, hypertension, body mass index, estimated glomerular filtration rate, and high-sensitivity C-reactive protein.

${ }^{*}$ Incidence rate per 1,000 person-years; ${ }^{\dagger}$ Excluded participants with use of lipid-lowering agent and adjusted for covariables in Model 3.

order to maximise the number of participants with lipids measurements before index year and to allow a longer follow-up period to capture the occurrence of stroke. Second, atrial fibrillation and diet were not examined, and thus, future study should determine whether these variables mediate the associations between lipids variability and stroke. Third, although we had carefully adjusted for lipid-lowering drug effects on lipids variability and the risk for stroke, residual confounding cannot be completely excluded. People with dyslipidemia are often encouraged by doctors to control blood pressure, weight, and restrict the intake of alcohol. Although various lipids measurements variability measured at three consecutive health examinations over 4 years, these time-varying behavioral variables, such as blood pressure and body weight, may modify serum lipids levels and have a favorable effect on the future stroke risk. Fourth, the associations of lipids profiles and stroke vary by stroke subtypes, ${ }^{9}$ however, we cannot further explore the potential effect of lipids variability on different subtypes of ischemic stroke. Because the information on the different subtypes of ischemic stroke was not available in the current study. Finally, the Kailuan Study was not designed to be nationally representative and all participants were recruited from Tangshan City; therefore, the generalizability of our findings to the whole Chinese adults needed to be cautious.

\section{Conclusions}

In conclusion, our findings suggest higher visit-to-visit variability in certain lipids measurements is associated with an increased risk for stroke and stroke type in a well-established community-based cohort study. High variability of HDL-C was independently associated with increased hemorrhagic stroke, whereas high variability of LDL-C increased the risk of ischemic stroke. Further interventional studies are warranted to deter- 
mine whether reducing variability in lipids can decrease the risk of stroke in the general population.

\section{Supplementary materials}

Supplementary materials related to this article can be found online at https://doi.org/10.5853/jos.2019.02075.

\section{Disclosure}

The authors have no financial conflicts of interest.

\section{Acknowledgments}

We thank all study participants, their relatives, the members of the survey teams at the 11 regional hospitals of the Kailuan Medical Group; and the project development and management teams at the Beijing Tiantan Hospital and the Kailuan Group.

This study was supported by the Young Elite Scientists Sponsorship Program by CAST (20180NRC001) and Beijing Municipal Administration of Hospitals Incubating Program (PX2020021).

\section{References}

1. Feigin $\mathrm{VL}$, Norrving $\mathrm{B}$, Mensah GA. Global burden of stroke. Circ Res 2017;120:439-448.

2. Wang W, Wang $D_{\text {, Liu }} H_{\text {, Sun }} H_{1}$ Jiang $B, R u X$, et al. Trend of declining stroke mortality in China: reasons and analysis. Stroke Vasc Neurol 2017;2:132-139.

3. Feigin $\mathrm{VL}$, Norrving $\mathrm{B}$, George $\mathrm{MG}$, Foltz JL, Roth $\mathrm{GA}$, Mensah GA. Prevention of stroke: a strategic global imperative. Nat Rev Neurol 2016;12:501-512.

4. Shahar $E_{1}$ Chambless LE, Rosamond WD, Boland LL, Ballantyne CM, McGovern PG, et al. Plasma lipid profile and incident ischemic stroke: the Atherosclerosis Risk in Communities (ARIC) study. Stroke 2003;34:623-631.

5. Tirschwell DL, Smith NL, Heckbert SR, Lemaitre RN, Longstreth WT Jr, Psaty BM. Association of cholesterol with stroke risk varies in stroke subtypes and patient subgroups. Neurology 2004;63:1868-1875.

6. Wang X, Dong Y, Qi X, Huang C, Hou L. Cholesterol levels and risk of hemorrhagic stroke: a systematic review and metaanalysis. Stroke 2013;44:1833-1839.

7. Xie L, Wu W, Chen J, Tu J, Zhou J, Qi X, et al. Cholesterol levels and hemorrhagic stroke risk in East Asian versus non-East Asian populations: a systematic review and meta-analysis. Neurologist 2017;22:107-115

8. Laloux $P$, Galanti L, Jamart J. Lipids in ischemic stroke sub- types. Acta Neurol Belg 2004;104:13-19.

9. Hindy G, Engström G, Larsson SC, Traylor M, Markus HS, Melander 0 , et al. Role of blood lipids in the development of ischemic stroke and its subtypes: a Mendelian randomization study. Stroke 2018;49:820-827.

10. Glasser SP, Mosher A, Howard G, Banach M. What is the association of lipid levels and incident stroke? Int J Cardiol 2016;220:890-894.

11. Pikula A, Beiser AS, Wang J, Himali JJ, Kelly-Hayes M, Kase CS, et al. Lipid and lipoprotein measurements and the risk of ischemic vascular events: Framingham Study. Neurology 2015;84: 472-479.

12. Bangalore S, Breazna A, DeMicco DA, Wun CC, Messerli FH; TNT Steering Committee and Investigators. Visit-to-visit lowdensity lipoprotein cholesterol variability and risk of cardiovascular outcomes: insights from the TNT trial. J Am Coll Cardiol 2015;65:1539-1548.

13. Waters DD, Bangalore $S$, Fayyad R, DeMicco DA, Laskey $R$, Melamed $S$, et al. Visit-to-visit variability of lipid measurements as predictors of cardiovascular events. J Clin Lipidol 2018;12:356-366

14. Boey E, Gay GM, Poh KK, Yeo TC, Tan HC, Lee CH. Visit-to-visit variability in LDL- and $\mathrm{HDL}$-cholesterol is associated with adverse events after ST-segment elevation myocardial infarction: a 5-year follow-up study. Atherosclerosis 2016;244:86-92.

15. Smit RA, Trompet S, Sabayan B, le Cessie S, van der Grond J, van Buchem MA, et al. Higher visit-to-visit low-density lipoprotein cholesterol variability is associated with lower cognitive performance, lower cerebral blood flow, and greater white matter hyperintensity load in older subjects. Circulation 2016;134:212-221.

16. Clark D 3rd, Nicholls SJ, St John J, Elshazly MB, Kapadia SR, Tuzcu EM, et al. Visit-to-visit cholesterol variability correlates with coronary atheroma progression and clinical outcomes. Eur Heart J 2018;39:2551-2558.

17. Kim MK, Han K, Kim HS, Park YM, Kwon HS, Yoon KH, et al. Cholesterol variability and the risk of mortality, myocardial infarction, and stroke: a nationwide population-based study. Eur Heart J 2017;38:3560-3566.

18. Kim MK, Han K, Park YM, Kwon HS, Kang G, Yoon KH, et al. Associations of variability in blood pressure, glucose and cholesterol concentrations, and body mass index with mortality and cardiovascular outcomes in the general population. Circulation 2018;138:2627-2637.

19. Wang $A$, Chen $S$, Wang $C$, Zhou $Y$, Wu $Y$, Xing $A$, et al. Resting heart rate and risk of cardiovascular diseases and allcause death: the Kailuan study. PLoS One 2014;9:e110985.

20. Xu Y, Li H, Wang A, Su Z, Yang G, Luo Y, et al. Association 
between the metabolically healthy obese phenotype and the risk of myocardial infarction: results from the Kailuan study. Eur J Endocrinol 2018;179:343-352.

21. Levey AS, Stevens LA, Schmid CH, Zhang YL, Castro AF 3rd, Feldman $\mathrm{HI}$, et al. A new equation to estimate glomerular filtration rate. Ann Intern Med 2009;150:604-612.

22. Stroke: 1989. Recommendations on stroke prevention, diagnosis, and therapy. Report of the WHO Task Force on Stroke and other Cerebrovascular Disorders. Stroke 1989;20:1407-1431.

23. Vidal-Petiot $E$, Stebbins $A$, Chiswell $K$, Ardissino D, Aylward $\mathrm{PE}$, Cannon $\mathrm{CP}$, et al. Visit-to-visit variability of blood pressure and cardiovascular outcomes in patients with stable coronary heart disease. Insights from the STABILITY trial. Eur Heart J 2017;38:2813-2822.

24. Wang A, Liu X, Xu J, Han X, Su Z, Chen S, et al. Visit-to-visit variability of fasting plasma glucose and the risk of cardiovascular disease and all-cause mortality in the general population. J Am Heart Assoc 2017;6:e006757.

25. Bangalore $S$, Fayyad R, Laskey R, DeMicco DA, Messerli FH, Waters DD. Body-weight fluctuations and outcomes in coronary disease. N Engl J Med 2017;376:1332-1340.

26. Lim YM, Yang PS, Jang E, Yu HT, Kim TH, Uhm JS, et al. Body mass index variability and long-term risk of new-onset atrial fibrillation in the general population: a Korean nationwide cohort study. Mayo Clin Proc 2019;94:225-235.

27. Lee SH, Han K, Cho H, Park YM, Kwon HS, Kang G, et al. Vari- ability in metabolic parameters and risk of dementia: a nationwide population-based study. Alzheimers Res Ther 2018; 10:110.

28. Kim MK, Han K, Koh ES, Kim HS, Kwon HS, Park YM, et al. Variability in total cholesterol is associated with the risk of end-stage renal disease: a nationwide population-based study. Arterioscler Thromb Vasc Biol 2017;37:1963-1970.

29. Yaghi S, Elkind MS. Lipids and cerebrovascular disease: research and practice. Stroke 2015;46:3322-3328.

30. Kim YD, Jung YH, Saposnik G. Traditional risk factors for stroke in East Asia. J Stroke 2016;18:273-285.

31. Lee JS, Chang PY, Zhang Y, Kizer JR, Best LG, Howard BV. Triglyceride and HDL-C dyslipidemia and risks of coronary heart disease and ischemic stroke by glycemic dysregulation status: the Strong Heart Study. Diabetes Care 2017;40:529-537.

32. Bardini G, Innocenti M, Rotella CM, Giannini S, Mannucci E. Variability of triglyceride levels and incidence of microalbuminuria in type 2 diabetes. J Clin Lipido/ 2016;10:109-115.

33. Wang A, Dai L, Su Z, Chen S, Li J, Wu S, et al. Proteinuria and risk of stroke in patients with hypertension: the Kailuan cohort study. J Clin Hypertens (Greenwich) 2018;20:765-774.

34. Wang A, Liu X, Su Z, Chen S, Zhang N, Wu S, et al. Two-year changes in proteinuria and the risk of stroke in the Chinese population: a prospective cohort study. J Am Heart Assoc 2017;6:e006271. 
Supplementary Table 1. Hazard ratios and 95\% confidence intervals of incident stroke by quartiles of total cholesterol variability*

\begin{tabular}{|c|c|c|c|c|c|c|}
\hline Variable & Quartile 1 & Quartile 2 & Quartile 3 & Quartile 4 & Per-SD increase & $P_{\text {trend }}$ \\
\hline \multicolumn{7}{|c|}{ Total stroke } \\
\hline SD & 1.00 & $1.07(0.91-1.26)$ & $0.92(0.77-1.09)$ & $1.14(0.97-1.35)$ & $1.04(0.98-1.10)$ & 0.337 \\
\hline VIM & 1.00 & $1.02(0.87-1.21)$ & $1.01(0.85-1.19)$ & $1.08(0.92-1.28)$ & $1.04(0.98-1.10)$ & 0.411 \\
\hline ARV & 1.00 & $0.99(0.83-1.17)$ & $0.94(0.79-1.11)$ & $1.12(0.95-1.33)$ & $1.04(0.98-1.10)$ & 0.241 \\
\hline \multicolumn{7}{|c|}{ Ischemic stroke } \\
\hline SD & 1.00 & $1.12(0.93-1.34)$ & $0.92(0.77-1.12)$ & $1.22(1.02-1.46)$ & $1.05(0.99-1.12)$ & 0.135 \\
\hline VIM & 1.00 & $1.05(0.88-1.26)$ & $1.02(0.85-1.23)$ & $1.15(0.96-1.37)$ & $1.06(1.00-1.13)$ & 0.179 \\
\hline ARV & 1.00 & $1.02(0.85-1.23)$ & $0.98(0.82-1.18)$ & $1.16(0.97-1.39)$ & $1.05(0.99-1.16)$ & 0.149 \\
\hline \multicolumn{7}{|c|}{ Hemorrhagic stroke } \\
\hline SD & 1.00 & $0.84(0.54-1.30)$ & $0.85(0.54-1.32)$ & $0.74(0.47-1.18)$ & $0.89(0.74-1.07)$ & 0.233 \\
\hline VIM & 1.00 & $0.87(0.56-1.34)$ & $0.87(0.56-1.35)$ & $0.71(0.44-1.13)$ & $0.89(0.74-1.07)$ & 0.174 \\
\hline ARV & 1.00 & $0.91(0.59-1.41)$ & $0.59(0.36-0.97)$ & $1.00(0.65-1.55)$ & $0.95(0.80-1.13)$ & 0.615 \\
\hline
\end{tabular}

SD, standard deviation; VIM, variability independent of the mean; ARV, average real variability.

*Adjusted for sex, age, mean total cholesterol level, education, income, smoking status, drinking status, physical activity, diabetes, hypertension, body mass index, estimated glomerular filtration rate, and high-sensitivity C-reactive protein.

Supplementary Table 2. Hazard ratios and 95\% confidence intervals of incident stroke by quartiles of triglycerides variability*

\begin{tabular}{|c|c|c|c|c|c|c|}
\hline Variable & Quartile 1 & Quartile 2 & Quartile 3 & Quartile 4 & Per-SD increase & $P_{\text {trend }}$ \\
\hline \multicolumn{7}{|c|}{ Total stroke } \\
\hline SD & 1.00 & $1.09(0.92-1.30)$ & $1.07(0.89-1.27)$ & $1.03(0.84-1.26)$ & $1.00(0.92-1.08)$ & 0.813 \\
\hline VIM & 1.00 & $0.99(0.84-1.16)$ & $0.90(0.76-1.06)$ & $0.93(0.78-1.10)$ & $0.98(0.92-1.04)$ & 0.233 \\
\hline ARV & 1.00 & $1.14(0.96-1.36)$ & $1.05(0.88-1.26)$ & $1.05(0.86-1.28)$ & $1.00(0.92-1.08)$ & 0.827 \\
\hline \multicolumn{7}{|c|}{ Ischemic stroke } \\
\hline SD & 1.00 & $1.08(0.89-1.30)$ & $1.03(0.85-1.24)$ & $1.03(1.83-1.28)$ & $0.98(0.90-1.07)$ & 0.876 \\
\hline VIM & 1.00 & $0.96(0.81-1.15)$ & $0.88(0.74-1.05)$ & $0.91(0.76-1.08)$ & $0.97(0.91-1.04)$ & 0.174 \\
\hline ARV & 1.00 & $1.16(0.96-1.40)$ & $1.16(0.88-1.28)$ & $1.06(0.85-1.31)$ & $0.97(0.90-1.06)$ & 0.806 \\
\hline \multicolumn{7}{|c|}{ Hemorrhagic stroke } \\
\hline SD & 1.00 & $1.16(0.74-1.81)$ & $1.03(0.64-1.65)$ & $0.77(0.42-1.41)$ & $1.08(0.82-1.41)$ & 0.471 \\
\hline VIM & 1.00 & $0.91(0.58-1.42)$ & $0.85(0.54-1.33)$ & $0.85(0.53-1.35)$ & $0.96(0.81-1.14)$ & 0.440 \\
\hline ARV & 1.00 & $1.16(0.74-1.80)$ & $0.93(0.58-1.50)$ & $0.81(0.45-1.45)$ & $1.12(0.88-1.43)$ & 0.410 \\
\hline
\end{tabular}

SD, standard deviation; VIM, variability independent of the mean; ARV, average real variability.

*Adjusted for sex, age, mean triglycerides level, education, income, smoking status, drinking status, physical activity, diabetes, hypertension, body mass index, estimated glomerular filtration rate, and high-sensitivity C-reactive protein. 
Supplementary Table 3. Hazard ratios and 95\% confidence intervals of incident stroke by quartiles of high-density lipoprotein cholesterol variability*

\begin{tabular}{|c|c|c|c|c|c|c|}
\hline Variable & Quartile 1 & Quartile 2 & Quartile 3 & Quartile 4 & Per-SD increase & $P_{\text {trend }}$ \\
\hline \multicolumn{7}{|c|}{ Total stroke } \\
\hline SD & 1.00 & $1.10(0.93-1.30)$ & $1.17(0.99-1.39)$ & $1.21(1.02-1.42)$ & $1.05(0.99-1.12)$ & 0.012 \\
\hline VIM & 1.00 & $1.10(0.93-1.30)$ & $1.17(0.99-1.39)$ & $1.21(1.02-1.42)$ & $1.07(1.02-1.13)$ & 0.021 \\
\hline ARV & 1.00 & $0.85(0.71-1.00)$ & $1.10(0.93-1.29)$ & $1.08(0.91-1.28)$ & $1.05(0.99-1.11)$ & 0.092 \\
\hline \multicolumn{7}{|c|}{ Ischemic stroke } \\
\hline SD & 1.00 & $1.14(0.95-1.37)$ & $1.30(1.08-1.56)$ & $1.26(1.04-1.52)$ & $1.06(1.00-1.13)$ & 0.008 \\
\hline VIM & 1.00 & $1.09(0.90-1.31)$ & $1.18(0.98-1.41)$ & $1.23(1.03-1.47)$ & $1.09(1.03-1.15)$ & 0.015 \\
\hline ARV & 1.00 & $0.90(0.75-1.09)$ & $1.14(0.95-1.36)$ & $1.12(0.93-1.35)$ & $1.06(1.00-1.12)$ & 0.063 \\
\hline \multicolumn{7}{|c|}{ Hemorrhagic stroke } \\
\hline SD & 1.00 & $1.16(0.75-1.79)$ & $0.89(0.55-1.42)$ & $0.82(0.50-1.35)$ & $0.93(0.78-1.11)$ & 0.296 \\
\hline VIM & 1.00 & $1.25(0.80-1.94)$ & $0.98(0.61-1.56)$ & $0.91(0.57-1.46)$ & $0.91(0.77-1.08)$ & 0.487 \\
\hline ARV & 1.00 & $0.48(0.30-0.79)$ & $0.75(0.49-1.16)$ & $0.69(0.44-1.10)$ & $0.89(0.74-1.08)$ & 0.232 \\
\hline
\end{tabular}

SD, standard deviation; VIM, variability independent of the mean; ARV, average real variability.

*Adjusted for sex, age, mean high-density lipoprotein cholesterol level, education, income, smoking status, drinking status, physical activity, diabetes, hypertension, body mass index, estimated glomerular filtration rate, and high-sensitivity C-reactive protein.

Supplementary Table 4. Hazard ratios and 95\% confidence intervals of incident stroke by quartiles of low-density lipoprotein cholesterol variability*

\begin{tabular}{|c|c|c|c|c|c|c|}
\hline Variable & Quartile 1 & Quartile 2 & Quartile 3 & Quartile 4 & Per-SD increase & $P_{\text {trend }}$ \\
\hline \multicolumn{7}{|c|}{ Total stroke } \\
\hline SD & 1.00 & $0.96(0.80-1.14)$ & $0.98(0.82-1.17)$ & $1.10(0.93-1.30)$ & $1.04(0.99-1.10)$ & 0.224 \\
\hline VIM & 1.00 & $1.00(0.84-1.20)$ & $0.95(0.79-1.14)$ & $1.12(0.95-1.33)$ & $1.04(0.99-1.10)$ & 0.223 \\
\hline ARV & 1.00 & $0.95(0.79-1.13)$ & $1.01(0.85-1.20)$ & $1.02(0.86-1.21)$ & $1.02(0.97-1.08)$ & 0.663 \\
\hline \multicolumn{7}{|c|}{ Ischemic stroke } \\
\hline SD & 1.00 & $0.91(0.75-1.10)$ & $0.91(0.75-1.10)$ & $1.01(0.84-1.21)$ & $1.02(0.96-1.10)$ & 0.843 \\
\hline VIM & 1.00 & $0.96(0.79-1.15)$ & $0.88(0.73-1.07)$ & $1.03(0.86-1.23)$ & $1.02(0.96-1.08)$ & 0.866 \\
\hline ARV & 1.00 & $0.90(0.74-1.09)$ & $0.95(0.79-1.14)$ & $0.94(0.78-1.12)$ & $1.00(0.94-1.06)$ & 0.621 \\
\hline \multicolumn{7}{|c|}{ Hemorrhagic stroke } \\
\hline SD & 1.00 & $1.62(0.92-2.84)$ & $2.05(1.19-3.53)$ & $2.31(1.19-3.53)$ & $1.21(1.06-1.37)$ & 0.001 \\
\hline VIM & 1.00 & $1.63(0.93-2.87)$ & $2.02(1.17-3.49)$ & $2.37(1.39-4.01)$ & $1.21(1.07-1.37)$ & 0.001 \\
\hline ARV & 1.00 & $1.46(0.84-2.52)$ & $1.89(1.12-3.18)$ & $1.98(1.19-3.32)$ & $1.15(1.01-1.37)$ & 0.006 \\
\hline
\end{tabular}

SD, standard deviation; VIM, variability independent of the mean; ARV, average real variability.

*Adjusted for sex, age, mean low-density lipoprotein cholesterol level, education, income, smoking status, drinking status, physical activity, diabetes, hypertension, body mass index, estimated glomerular filtration rate, and high-sensitivity C-reactive protein. 